\title{
Abundancia de Marmosa xerophila y Marmosa robinsoni (Didelphimorphia: Didelphidae) en la mina El Cerrejón, La Guajira, Colombia
}

\author{
Orlando Padilla-Rivera \\ Departamento de Biología, Universidad del Atlántico. Barranquilla-Colombia orlandopadillarivera@ gmail.com
}

\section{Resumen}

Como parte de las acciones de monitoreo de la fauna silvestre de la mina El Cerrejón, se evaluó la abundancia de las especies Marmosa xerophila y Marmosa robinsoni en tres sectores diferentes (bosque seco secundario del área de La Estrella, bosque ripario intervenido del arroyo Tabaco y arbustos restaurados en el área de Rehabilitación-Manantial) mediante captura con trampas Sherman. Se consideró el éxito de captura como descriptor de la abundancia. Marmosa xerophila fue poco abundante $(\mathrm{n}=2)$ y restringida a la zona de arbustos restaurados, mientras que la especie Marmosa robinsoni se halló con mayor abundancia $(\mathrm{n}=21)$ con presencia en todas las zonas muestreadas. Se considera que Marmosa robinsoni es una especie abundante debido a que puede ocupar hábitats transformados como los presentes en el área de estudio y que Marmosa xerophila está en desventaja ante la trasformación del hábitat.

Palabras clave: La Guajira; Éxito de captura; Abundancia; Bosque xerófilo.

\section{Abstract}

As part of monitoring actions for wildlife in El Cerrejón coalmine, an assessment of the abundance of Marmosa xerophila and Marmosa robinsoni was done in three different areas (La Estrella tropical dry forest secondary growth, Tabaco creek disturbed riparian forest, and artificially restored bushes from restoration-Manantial area) using Sherman traps. Capture success was considered as a descriptor of abundance. Marmosa xerophila was found with low abundance $(\mathrm{n}=2)$ and restricted to restored bush areas, meanwhile, Marmosa robinsoni was found in higher abundance $(\mathrm{n}=21)$ and was present in all sampled areas. Marmosa robinsoni is considered dominant in terms of abundance because can occupy in disturbed habitats like those in the study area and Marmosa xerophila is considered to be strongly affected by habitat transformation.

Key words: La Guajira; Capture success; Abundance; Xerophitic forest.

\section{Introducción}

Entre las especies del género Marmosa (Gray 1821) conocidas en Colombia, Marmosa robinsoni está presente en Panamá, Colombia, Venezuela y las islas de Trinidad, Tobago y Granada (Eisenberg 1989, Rossi et al. 2010, Gutiérrez et al. 2012, Gutiérrez et al. 2014b, Pérez-Hernández et al. 2016a). En contraste, Marmosa xerophila es endémica de los hábitats áridos y bosques secos en un área restringida en el norte de Colombia (departamento de La Guajira) y el noroccidente de Venezuela en los estados de Zulia y Falcón (Eisenberg 1989, Thielen et al. 1997a, b). M. xerophila está categorizada como Vulnerable por la Lista Roja de Especies Amenazadas de la UICN (Pérez-Hernández et al. 2016b), mientras que M. robinsoni está catalogada en Preocupación menor (Pérez-Hernández et al. 2016a).

Así mismo, Marmosa xerophila y M. robinsoni son dos especies hermanas (Voss et al. 2014) que son dependientes de condiciones de microhábitat como la estructura y composición de la vegetación en lo referente a su abundancia y además tienen requerimientos ecológicos similares como la dieta (Thielen et al. 1997a). También, es importante resaltar que estas dos especies encuentran en la mina de El Cerrejón una zona de sobreposición de su distribución.

En este contexto, la compañía minera planea continuar sus operaciones de explotación por lo menos dos décadas más en esta área predominantemente dominada por bosque seco tropical el cual es un hábitat muy vulnerable a las perturbaciones humanas, circunstancia que derivó en un programa de rehabilitación de dicho hábitat que comenzó en 2004 (Domínguez-Haydar \& Ambrecht 2011). Consecuentemente, este estudio se desarrolló con el objetivo de conocer la abundancia y su representación en los hábitats presentes en la mina El Cerrejón como insumo de monitoreo de las acciones de restauración en el hábitat en el proyecto minero con miras a conocer la dinámica de ambas especies en estas circunstancias. 


\section{Materiales y métodos}

Área de estudio

El estudio se realizó dentro del área de influencia de la mina El Cerrejón ubicada entre los municipios de Albania, Barrancas y Hatonuevo, departamento de La Guajira (Figura 1). La zona de estudio se encuentra en la zona de vida de bosque seco tropical y matorral xerófilo tropical (Holdridge 1967); la distribución promedio de precipitación es bimodal con una precipitación media anual $840 \mathrm{~mm}$ y una temperatura media anual de $28,5^{\circ} \mathrm{C}$ (Domínguez-Haydar \& Ambrecht 2011).

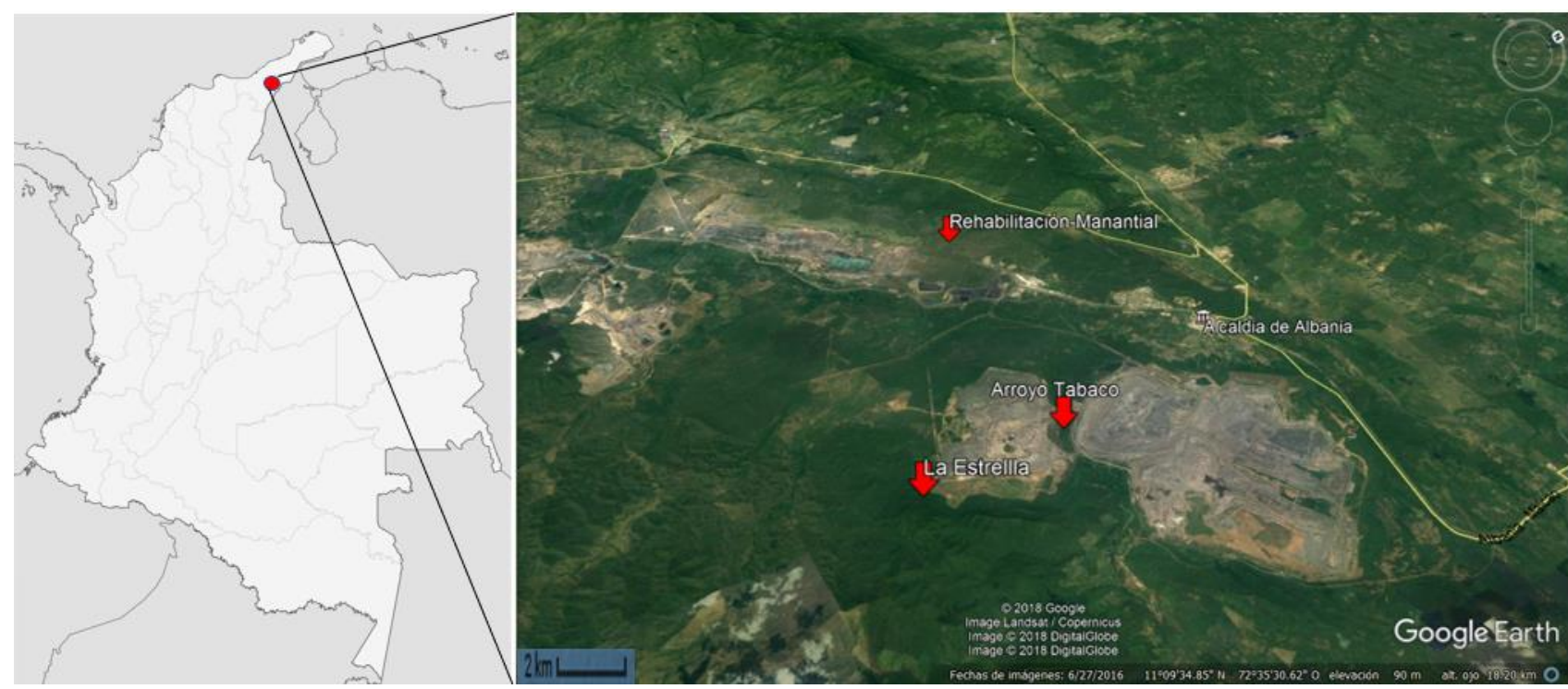

Figura 1. Ubicación de los sectores de muestreo en el complejo minero El Cerrejón. Google Earth Pro V 7.3.1 (mayo 26, 2018).

Específicamente, dentro del área de estudio se identificaron tres tipos de hábitats predominantes y se ubicaron asimismo tres localidades de muestreo: i) área rehabilitación - Manantial que consta de matorrales abiertos $(11,061785 \mathrm{~N}$, $72,41273 \mathrm{~W})$ a $174 \mathrm{msnm}$, ii) ronda riparia asociada al arroyo Tabaco (11,06245N, -72,34336W) a $112 \mathrm{msnm}$, y iii) sector "La Estrella" que incluye bosque xerófilo menos alterado (11,05439N, -72,33476W) a $210 \mathrm{msnm}$.

\section{Métodos}

En cada una de las tres localidades se instalaron 40 trampas Sherman; en cada sitio 20 trampas fueron ubicadas en el suelo y las restantes en ramas de arbustos y matorrales a $2 \mathrm{~m}$ de altura (de manera intercalada), a lo largo de dos transectos con una extensión aproximada de $500 \mathrm{~m}$ cada uno. Las trampas fueron cebadas a diario con mantequilla de maní, avena en hojuelas y maíz. Cada trampa fue ubicada a una distancia de entre 25 m y 35 m entre sí, abarcando una extensión aproximada de 1 ha (Figura 1). Todas las trampas estuvieron activas durante 19 noches en total y se estableció el éxito de captura según Romero et al. (2007) quienes definen que el éxito de trampeo es igual al porcentaje de trampas con animales capturados.

Se consideró la temporada seca para este estudio por ser una época crítica para la consecución de alimentos favoreciendo así el éxito de capturas (Thielen 1997b), y también porque es posible que en la época de lluvias los torrentes repentinos y las precipitaciones laven el cebo y cierren las trampas (Romero et al. 2007). Cuando se presentaron capturas, los animales fueron depositados en bolsas de tela para marcaje con cortes en las orejas y posterior liberación. En cuanto a la identificación de las dos especies (Figura 2), éstas se diferencian por las marcas oculares reducidas, flancos y abdomen muy claros contrastantes con el manto marrón grisaceo en M. xerophila y la coloración dorsal y longitud del pelaje dorsal, que en M. robinsoni es de color amarillento y más largo. Otros caracteres adicionales para separar las especies son el patrón de coloración del pelaje ventral con banda gris basal generalizada en $M$. xerophila, en contraste con una banda basal sólo en los lados del vientre en $M$. robinsoni; este último es un carácter verificable en juveniles (Rodríguez-Mahecha et al. 2006, Gardner 2008, Rossi et al. 2010). 


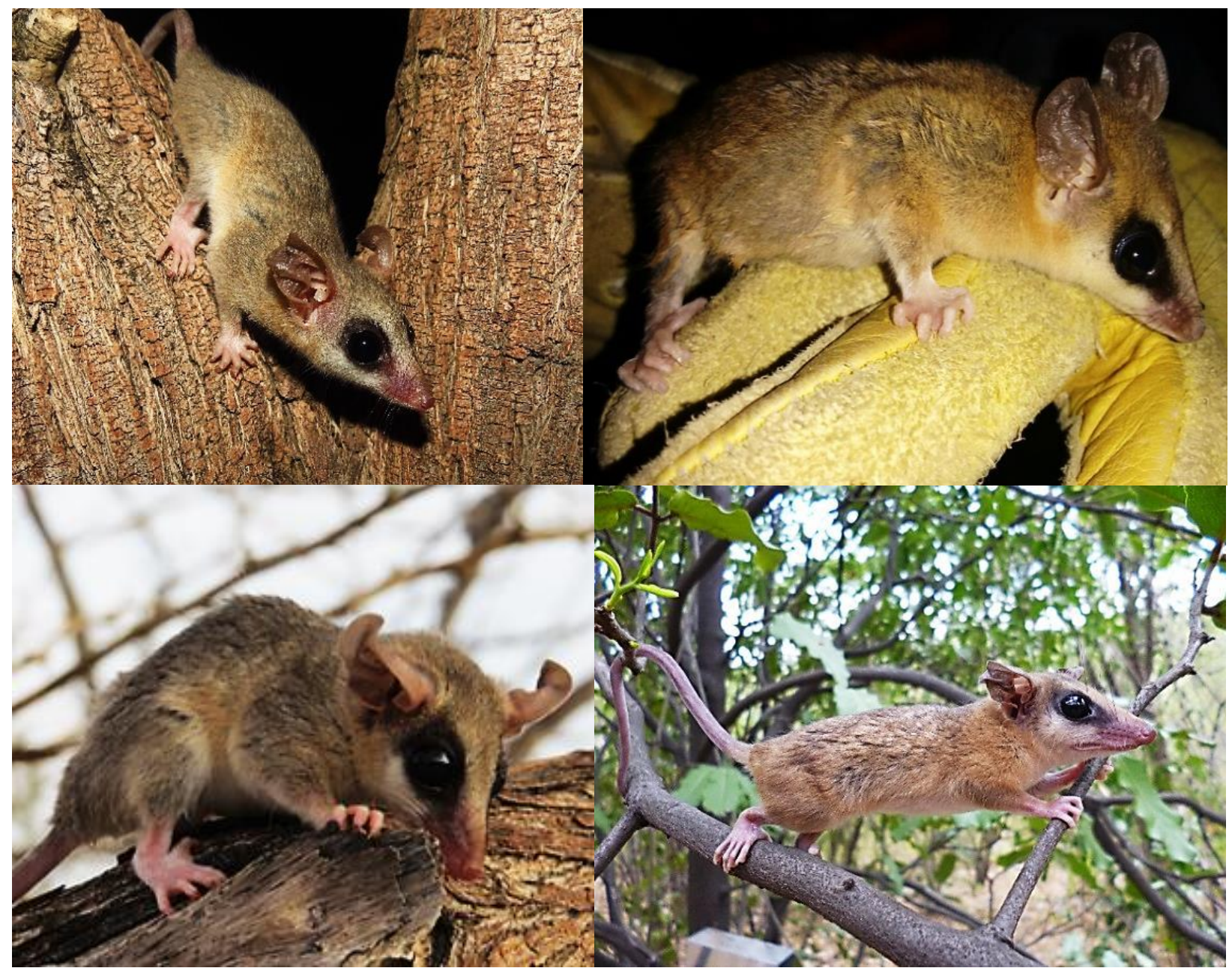

Figura 2. Individuos de Marmosa xerophila (izq.) y Marmosa robinsoni (der.) capturado en La Guajira, Colombia

\section{Resultados}

\section{Abundancia}

Durante 19 noches de monitoreo, 120 trampas activadas y un esfuerzo de captura total de 2.280 trampas/noche se capturaron 25 pequeños mamíferos. De estos 21 corresponden a individuos de M. robinsoni, mientras que M. xerophila registró dos individuos y las otras dos capturas correspondieron a Proechymis canicollis (Rodentia: Cricetidae).

Específicamente, en el sector de monitoreo arroyo Tabaco se capturaron nueve individuos, en el área de rehabilitaciónManantial se capturaron 13 individuos y en el área de La Estrella se capturaron tres individuos. El éxito de captura general fue del 19,16\% sólo para el género Marmosa; para cada especie fue 17,5\% para M. robinsoni y 1,6\% para $M$. xerophila en todas las áreas de muestreo. Para M. robinsoni en arroyo Tabaco fue del 22,5\%, mientras que en la zona rehabilitación fue del 27,5\% y en La Estrella del 7,5\%. El área donde se registró la captura de dos individuos de $M$. xerophila fue la zona de rehabilitación-Manantial con un éxito de captura del $5 \%$.

\section{Presencia en hábitats}

No fue posible establecer una asociación a un hábitat para M. xerophila debido a la escasez de capturas y que sólo se encontró en un área de rehabilitación caracterizada por arbustos xerófilos de sucesión temprana con proximidad a un cuerpo de agua léntico.

Paralelamente, la especie $M$. robinsoni registró presencia en todas las áreas muestreadas, con capturas en las tres localidades de monitoreo. La abundancia fue mayor en cercanías a cuerpos de agua $(n=20)$ aunque muy diferentes en cuanto la vegetación, como es el caso de arroyo Tabaco con su faja de bosque ribereño intervenido y área de 
rehabilitación-Manantial con sus arbustales abiertos, y menos abundante en el bosque seco del área de la Estrella con tres capturas.

\section{Discusión}

\section{Abundancia}

Estos resultados ponen en contexto la baja abundancia de la especie $M$. xerophila, la cual es indicadora de la vegetación xerófila tropical o bosque espinoso tropical (Handley \& Gordon 1979, Eisenberg 1989, Thielen et al. 1997a, b, Rodríguez-Mahecha et al. 2006, Lew et al. 2011). Aunque M. xerophila está adaptada a este tipo de hábitat, se observó con mínima abundancia respecto a su congénere. En cuanto a la estimación del éxito de captura, un valor mayor para $M$. robinsoni implica que se trata de la especie dominante en el periodo analizado, aspecto consecuente con su carácter generalista (Alvizú \& Aguilera 1998).

Adicionalmente, existe un vacío en los registros de M. xerophila en otros reportes de El Cerrejón desde 1982 a 2000 y también un vacío de registros en el área de rehabilitación-Manantial del 2005 y 2006 ya que se capturaron 12 individuos de esta especie en 6 sectores diferentes de la mina (Los Pozos, Tabaco, La Puente, Cerrejón, Paladines, Compensación y Calaguala) y ningún individuo en el área de rehabilitación (Fundación Hidrobiológica George Dahl 2006). Dichos estudios reflejan que la especie tiene una presencia más amplia que la observada en este estudio; igualmente, la especie M. robinsoni también presentó una distribución amplia, pero con mayores abundancias y ausente también en el área de rehabilitación (Fundación Hidrobiológica George Dahl 2006), lo que implicaría fluctuaciones en la presencia de la especie en el tiempo o una reciente colonización en el área cuya vegetación fue sembrada hace 24 años (DomínguezHaydar \& Ambrecht 2011, Báez \& Trujillo 2014).

La tendencia en el tiempo a la baja abundancia de M. xerophila puede deberse a factores como el acceso a recursos alimenticios (Thielen et al. 1997b) o refugios, limitado por la competencia sobre estos con M. robinsoni. La dificultad de acceder a fuentes de alimento puede asociarse a que ambas especies tienen una dieta muy afín y aprovechan los recursos de manera similar en las temporadas climáticas (invertebrados y frutos; Thielen et al. 1997a). Por otra parte, la baja abundancia de $M$. xerophila puede explicarse por un mecanismo de exclusión competitiva, debido a que $M$. xerophila prevalece en hábitats xerofíticos y $M$. robinsoni la reemplaza en habitas adyacentes más mesófilos en la península de Paraguaná al norte de Venezuela, región muy afín ecológicamente a La Guajira (Gutiérrez et al. 2014a). Estos reportes son congruentes con los datos obtenidos entre 2005 y 2006, que muestran la superioridad numérica de $M$. robinsoni sobre M. xerophila (Fundación hidrobiológica George Dahl 2006), sin embargo, las densidades de población en un estudio de campo sugieren variabilidad estacional de 6 a 20 individuos de M. xerophila por hectárea en hábitat favorable (Thielen et al. 1997c).

Puntualmente, en el caso de la especie $M$. xerophila se tiene evidencia que su supervivencia puede estar influenciada por la cercanía que tienen las crías al área vital de las madres (Thielen 1997 b, c); de esta manera, la familiaridad que tengan los individuos a su entorno inicialmente conocido es la estrategia de supervivencia concreta para esta especie (Thielen 1996). En contraste, M. robinsoni ha mostrado evidencia de territorialidad en hábitats áridos en Venezuela (Alvizu \& Aguilera 1998). En este contexto, M. xerophila tiene comprometida su permanencia en la zona por afectaciones del hábitat como la tala, la extracción de madera y la fragmentación. De esta manera, el manejo y la conservación del hábitat típico de M. xerophila (98\% bosque espinoso; Handley \& Gordon 1979) es de carácter crucial para la supervivencia de esta especie emblemática de la región.

Finalmente, es necesario monitorear la dinámica de abundancia y distribución en paisajes alterados entre estas especies mediante más muestreos que incluyan puntos previamente muestreados y a través de censos multitemporales con los que se puedan estimar la dinámica de estas especies con la intención de saber si la baja abundancia de M. xerophila es una tendencia hacia la extinción local y si obedece a reemplazo por competencia de su congénere M. robinsoni. Además, se requiere en conjunto un estudio en el cual se determine el factor limitante para la presencia y abundancia de la especie: competencia, degradación de hábitat, entre otros, y establecer un escenario que pronostique la presencia de la especie. Este escenario proveería elementos de juicio para que el esquema de intervención minero proyecte y priorice áreas de conservación y acciones que dirijan hacia la protección del hábitat de M. xerophila en la zona de influencia de la mina y así plantear acciones de acuerdo a su plan de intervención y cierre de la explotación.

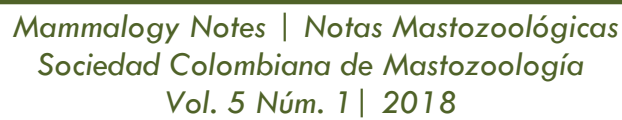




\section{Agradecimientos}

Este estudio es parte de las actividades de monitoreo de la biodiversidad del complejo minero Cerrejón en su momento a cargo de Lina Báez especialista en biodiversidad a quien agradezco su amable apoyo durante la fase de campo y sus observaciones; también agradezco a los biólogos Erhent Madariaga y Helí Arciniégas de Bioma Consultores por su acuciosa revisión del manuscrito base, a la bióloga Erika Humánez por sus comentarios en la versión inicial de este aporte y a Héctor Ramírez en la versión final del mismo.

\section{Referencias}

Alvizú, P. \& M. AguilerA. 1998. Uso del espacio de Marmosa robinsoni (Didelphidae: Marsupialia) en una zona xerófila de los Andes venezolanos. Ecotropicos 11(2): 81-92.

BÁEZ, L. \& F. Trujillo (eds.). 2014. Biodiversidad en Cerrejón. Carbones de Cerrejón, Fundación Omacha, Fondo para la Acción Ambiental y la Niñez. Bogotá, Colombia. 352.

CAughley, G. 1977. Analysis of vertebrate populations. John Wiley and Sons, New York, USA, 234.

DomínguEZ-HAYDAR, Y. \& I. ARMBRECHT. 2011. Response of ants and their seed removal in rehabilitation areas and forests at El Cerrejón Coal Mine in Colombia. Restoration Ecology 19(201): 178-184.

Fundación HidrobiológiCA GeORGE DAHL. 2006. Monitoreo de fauna en el valle del Cerrejón 2005-2006. Contrato con 00412005. Informe final.

GARDNER, L.A. 2008. American Marsupials. Pp 1-11 In Mammals of South America, Volume 1: Marsupials, Xenarthrans, Shrews, and Bats (Gardner LA, ed.) University of Chicago Press.

Google Earth Pro V 7.3.1. 2018. La Guajira, Colombia. $11^{\circ} 06^{\prime} 38.20^{\prime} \mathrm{S}, 72^{\circ} 34^{\prime} 52.49$ ” W, Eye alt 18,20 km. Digital Globe 2016. http://www.earth.google.com [mayo 26, 2018].

GuTIÉRREZ, E., et al. 2014a.Can biotic interactions cause allopatry? Niche models, competition, and distributions of South American mouse opossums. Ecography 37: 741-753.

GutiÉRREZ, E., et al. 2014b. Phylogeography of Marmosa robinsoni: insights into the biogeography of dry forest in northern South America. Journal of Mammalogy 95(6): 1175-1188.

Handley, C.O. JR. \& L.K. GoRdON. 1979. New species of mammals from Northern South America. Mouse Opossum, Genus Marmosa Gray. Pp 65-72 In: Vertebrate ecology in the Northern Neotropics (Eisenberg JF, ed.). Smithsonian Institution Press, Washington, D.C.

HoldridGE, L. R. 1967. Life Zone Ecology. Tropical Science Center. San José, Costa Rica.

PÉREZ-HernANDEZ, R. 2016a. Marmosa robinsoni. The IUCN Red List of Threatened Species 2016: e.T40506A22174162.

PÉREZ-HERnÁNDEZ, R., et al. 2016b. Marmosa xerophila. (errata version published in 2017) The IUCN Red List of Threatened Species 2016: e. T12815A115106154.

Romero-Almaraz, M., et al. 2007. Mamíferos pequeños: Manual de técnicas de captura, preparación preservación y estudio. Universidad Nacional Autónoma de México, Facultad de Ciencias, Instituto de Biología. Segunda edición, Navegantes de la Comunicación Gráfica, S. A. 203.

Rossi, R. V., et al. 2010. A revision of the Didelphid Marsupial Genus Marmosa Part 1. The Species in Tate's 'Mexicana' and 'Mitis' sections and other closely related forms. Bulletin of the American Museum of Natural History 334: 1-83.

ThIELEN, D.R. 1996. Ecología poblacional de Marmosa xerophila en un ecosistema semiárido del norte de Venezuela. Tesis de maestría. Postgrado en ecología tropical. Universidad de los Andes, Mérida, Venezuela. 233.

THIELEN, D.R., et al. 1997a. Population ecology of Marmosa xerophila in a semiarid ecosystem of northern Venezuela. Zoocriaderos 2(1): 1-19.

ThIELEN, D.R., et al. 1997b. Selección de microhabitat por Marmosa xerophila (Marsupalia: Didelphidae) en un ecosistema semiárido del norte de Venezuela. Ecotropicos 10(1): 21-28.

ThIELEN, D. R., et al. 1997c. Food availability and population dynamics of Marmosa xerophila Handley and Gordon 1979 (Marsupialia: Didelphidae). Zoocriaderos 2(1): 1-15.

THIELEN, D.R., et al. 2009. Rearing cycle and other reproductive parameters of the xero-phytic mouse opossum Marmosa xerophila (Didelphimorphia: Didelphidae) in the peninsula of Paraguana. Interciencia 34: 195-198.

Voss, R. S., et al. 2014. Phylogenetic relationships of mouse opossums (Didelphidae, Marmosa) with a revised subgeneric classification and notes on sympatric diversity. American Museum Novitates 3817: 1-27. 